\title{
Central Vein
}

National Cancer Institute

\section{Source}

National Cancer Institute. Central Vein. NCI Thesaurus. Code C32281.

The veins within the hepatic lobes that drain blood from the liver sinusoids into the hepatic vein. 